J. Oksanen and J. Tulkki, Fast $2 R$ regeneration by coherent laser amplifiers, IEEE Journal of Quantum Electronics 41, pp. 1075-1082 (2005).

(C) 2005] IEEE. Reprinted, with permission, from IEEE Journal of Quantum Electronics.

This material is posted here with permission of the IEEE. Such permission of the IEEE does not in any way imply IEEE endorsement of any of Helsinki University of Technology's products or services. Internal or personal use of this material is permitted. However, permission to reprint/republish this material for advertising or promotional purposes or for creating new collective works for resale or redistribution must be obtained from the IEEE by writing to pubs-permissions@ieee.org.

By choosing to view this document, you agree to all provisions of the copyright laws protecting it. 


\title{
Fast 2R Regeneration by Coherent Laser Amplifiers
}

\author{
Jani Oksanen and Jukka Tulkki
}

\begin{abstract}
A new device concept based on coherent processing of optical signals and allowing generation of ultrafast all-optical functions is introduced. The device is modeled by rate equations and its functionality is demonstrated by studying the re-amplification and re-shaping ( $2 R$ ) regeneration of a 30-GHz signal in detail. The main components of the regenerator are phase-locked lasers, optical isolators and band pass filters. Small signal analysis of the model predicts that bandwidths in excess of $100 \mathrm{GHz}$ might be attainable. The technological feasibility of the device is also discussed.
\end{abstract}

Index Terms-Coherent optics, function generation, optical isolators, optical regeneration, semiconductor laser.

\section{INTRODUCTION}

$\mathbf{T}$ HE TRANSITION from electrical to optical domain in telecommunication industries started out by deploying optical fibers and fiber optical amplifiers in the long haul network backbone. These components were like tailor-made for transferring data over long distances. However, expanding optical technologies to access and metropolitan area networks has not progressed as smoothly. The common reason for both the early success of the communication optics, and the difficulties found later in approaching the end user is the linearity of optical processes. Therefore, developing practical purely optical components enabling fast switching, logic, and other nonlinear functions has proven a difficult challenge.

This paper presents a new device concept that can be used to realize arbitrary all-optical functions. The key elements in the device operation are phase-locked lasers, which are modulated by optical signals. The emphasis of the paper is on realizing a re-amplification and re-shaping $(2 \mathrm{R})$ regenerator, required in preserving the data integrity on long transmission paths.

Regenerators operating in purely optical domain and based on different operation principles ranging from nonlinear fibers to semiconductor optical amplifiers are being actively pursued [1], [2]. Devices based on gain-clamped semiconductor optical amplifiers and injection-locked lasers have been demonstrated as well [3], [4]. They rely on the saturation of laser fields, and consequently their operating frequency is limited by the relaxation oscillations and ultimately by the turn on delay of the lasers.

In the present construction the relaxation oscillations can be suppressed and the device needs not be driven to saturation. Therefore, the modulation speed is not limited by the same mechanisms as in the other semiconductor regenerators based on the use of lasers. As expected, the proposed device is more

Manuscript received March 23, 2005.

The authors are with the Department of Electrical and Communications Engineering, Laboratory of Computational Engineering, Helsinki University of Technology, Helsinki, FIN-02015, Finland (e-mail: Jani.oksanen@1ce.hut.fi; Jukka.tulkki@hut.fi).

Digital Object Identifier 10.1109/JQE.2005.851244 complex than the previously introduced regenerators relying on saturation effects.

The paper is organized as follows. Section II describes the model used in simulations. In Section III, the structure and parameters needed to realize the regenerator are presented. The results of the simulations are given and discussed in Section IV, and Section V concludes the paper. In the Appendix a small signal approximation is used to find the limits where no relaxation oscillations take place and to determine the approximate time constants of a single laser.

\section{THEORY}

This section presents the model of a device composed of several forward coupled lasers. Section II-A gives a qualitative presentation of the device, starting with the steady-state response of a single laser and expanding the concept to a network of several lasers, described qualitatively and mathematically. Section II-B quantifies the operation of a single laser by introducing a rate equation model describing the dynamical evolution of the carrier density and the fields in the laser. Section II-C combines the single laser rate equations into a matrix equation by introducing coupling matrices and bias vectors to describe the interaction between the lasers. The actual material and device parameters of the regenerator are discussed in Section III.

\section{A. General Operation Principle}

The steady-state output of gain clamped laser amplifiers consists of two signals at different frequencies: the output at the frequency of the signal mode (electric field $E_{s}^{\text {out }}$, power $P_{s}^{\text {out }}$ ) and the output at the frequency of the laser mode $\left(E_{L}^{\text {out }}, P_{L}^{\text {out }}\right)$. In ideal operation the input-output responses are linear $\left(E_{s}^{\text {out }} \propto\right.$ $E_{s}^{\text {in }}, P_{s}^{\text {out }} \propto P_{s}^{\text {in }}, P_{L}^{\text {out }} \propto 1-c_{L} P_{s}^{\text {in }}$, where $c_{L}$ is a constant $)$, except in terms of the electric field $E_{L}^{\text {out }} \propto \sqrt{1-c_{L}\left|E_{s}^{\text {in }}\right|^{2}}$, which is nonlinear. In addition to this nonlinear relation, the device construction makes use of linear operations on electric fields by lossy waveguides (multiplication by a constant $\leq 1$ ) and by waveguide interferometers (addition of coherent electric fields with constructive phases and subtraction with destructive phases). The objective of the device is to generate a desired transfer function by using coherent light, linear waveguides and the nonlinearity of the electric field. Due to the nonlinear electric field dependence, this can be done without driving the lasers to saturation. The transfer function generation process resembles the mathematical methods where some basis functions (outputs of the lasers) are combined (by interferometers) to create a more complex function (the output). An example of a possible realization is shown in Fig. 1.

The qualitative physical operation of the device shown in Fig. 1 is the following. First coherent reference signals at wavelengths $\lambda_{1}$ and $\lambda_{2}$ are generated by two master lasers (not shown 


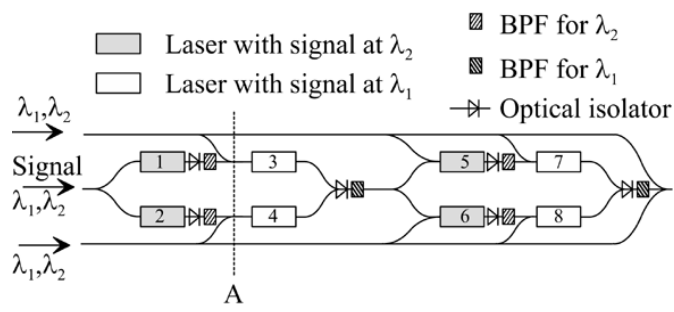

Fig. 1. Schematic structure of the regenerator. The lasers are of two different types with the signal and cavity mode frequency alternating between consecutive stages. The structure also makes use of bandpass filters (BPF) and optical isolators. Additional band pass filters and loss elements, that are not drawn to the picture, are also needed in the waveguides injecting the phase locking and biasing fields. The numbers inside the lasers denote the corresponding row and element numbers in the coupling matrix and other vectors. The input and output signal wavelengths are $\lambda_{1}$.

in the figure). The input signal needs to have the same frequency and phase as $\lambda_{1}$, and if this is not the case, a conversion is necessary. Next a small bias signal at $\lambda_{2}$ is injected to lasers 1 and 2 to lock their phases (the lasers operate at $\lambda_{2}$ ). Also appropriately selected combinations of the signal (at $\lambda_{1}$ ) and a bias signal at $\lambda_{1}$ are injected to lasers 1 and 2 . The waveguides are assumed to have different absorption coefficients, and therefore the amplitudes of the signal and bias signals injected in the lasers can be individually adjusted. For simplicity these effects are not discussed further in this qualitative description. The outputs of the lasers 1 and 2 are led to optical isolators to prevent reflections and backward propagation, and to band pass filters that let $\lambda_{2}$ through.

Now the signal information is contained at the wavelength $\lambda_{2}$, originating from lasers 1 and 2 . These new signals at plane A in Fig. 1 are again made to interfere with the appropriately adjusted bias signals from the master laser at $\lambda_{2}$ and fed to lasers 3 and 4 along with the phase locking signal, now at $\lambda_{1}$, which is the lasing mode of lasers 3 and 4 . The same procedure is repeated through the device, and the final output at $\lambda_{1}$ is a function determined by the bias signals, the loss coefficients of the waveguides and the general configuration of the lasers.

The normalized electric field $y_{m}$ at the output of laser $m$ of an arbitrary structure composed of $M$ phase-locked lasers connected by a network of waveguides and with no optical feedback between the lasers can be written in a recursive form

$$
y_{m}=\Re \sqrt{1-\left(B_{m}^{\mathrm{in}}+\Sigma_{n=0}^{m-1} a_{m, n}^{\mathrm{in}} y_{n}\right)^{2}} .
$$

Here $\Re$ denotes the real part, $B_{m}^{\text {in }}$ is the constant bias signal added to the input of the laser $m$ and $a_{m, n}^{\text {in }}$ are coefficients determined by the waveguide network. The original input signal $x$ is included by setting the term $y_{0}$ to $y_{0}=x$. All the coefficients and parameters are real. The final output $Y$ after the $M$ th laser can be written as $Y=B^{\text {out }}+\sum_{n=0}^{M} a_{n}^{\text {out }} y_{n}$, where $B^{\text {out }}$ and $a_{n}^{\text {out }}$ are an appropriately selected bias signal and waveguide coefficients, respectively.

The nonlinearity of a laser is enhanced the closer it gets to saturation. However, to maintain high-speed operation the operating point must not be set too close to saturation. The nonlinearity can be harnessed, for example, by the circuit shown in Fig. 1 to implement the regenerator studied later in this paper.
The coherent nature of the device intrinsically implies that there is a master laser providing the reference phases for the system. Because the phase of the input signal is not well defined at times of no input, the input signal must be converted to a suitable wavelength and phase, given by a master laser. This conversion is assumed to take place, but for simplicity not included in the model. In other words, the input signal is assumed to be a coherent signal, even if the original signal was chaotic.

\section{B. Model of a Single Laser}

A standard way of describing the basic building block of the device, the semiconductor laser, is the rate equations. They enable the study of many aspects of laser operation, including but not limited to relaxation oscillations, turn on delay, chirp, noise properties and phase locking.

A single laser with external light injection to a remote cavity mode can be described by rate equations accounting for the carrier density $n$ (normalized to the photon cavity volume), the complex electric field at the frequency of the cavity mode $E_{L}$ and at the signal mode $E_{s}$

$$
\begin{aligned}
\frac{d n}{d t} & =\frac{I}{q V}-\frac{n}{\tau}-v \xi G_{L}(n)\left|E_{L}\right|^{2}-v \xi G_{s}(n)\left|E_{s}\right|^{2} \\
\frac{d E_{L}}{d t} & =\left[\frac{v}{2}\left(G_{L}(n)-\alpha_{L}\right)+i \Delta \omega_{L}(n)\right] E_{L}+\frac{v}{2 L} E_{L}^{\mathrm{ext}} \\
\frac{d E_{s}}{d t} & =\left[\frac{v}{2}\left(G_{s}(n)-\alpha_{s}\right)+i \Delta \omega_{s}(n)\right] E_{s}+\frac{v}{2 L} E_{s}^{\mathrm{ext}} .
\end{aligned}
$$

These equations are based on well-known models of the phase locking behavior of semiconductor lasers, with an additional equation for the signal mode and some modifications in notation [5]-[9]. Both electric fields $E_{L}$ and $E_{s}$ are considered as the slowly varying complex amplitudes of the coherent traveling waves with the steady-state cavity mode frequency removed, i.e. the total photon density $n_{p h}=\xi\left|E^{+}\right|^{2} / 2+\xi\left|E^{-}\right|^{2} / 2=\xi|E|^{2}$, where $\xi / 2$ is the conversion factor between photon density and the square of electric field magnitude $\left(\xi=\sqrt{\varepsilon \mu^{-1}} /(\hbar \omega v)\right)$ and $E^{+}$and $E^{-}$are the complex amplitudes of the forward and backward propagating fields, which are both approximately equal to $E$. The symbols appearing in the equations are the effective injection current $(I)$, the volume of the optical cavity $(V)$, carrier lifetime without stimulated emission taken into account $(\tau)$, the speed of light in the medium $(v)$, the modal gain and loss of the mode $i \in\{L, s\}\left(G_{i}\right.$ and $\left.\alpha_{i}\right)$, the deviation of the cavity resonance of the mode $i$ from its steady-state value $\left(\Delta \omega_{i}\right)$, the length of the cavity $(L)$, and the external injection into the mode $\left(E_{i}^{\text {ext }}\right)$.

The gain in (2)-(4) is modeled by

$$
G_{i}(n)=G_{i}^{\max } \frac{n-n_{i}^{0}}{n+n_{i}^{0}}
$$

where $G_{i}^{\max }$ is the maximum modal gain and $n_{i}^{0}$ is the transparency carrier density. The change in the frequency of the cavity mode $i$ is given by

$$
\Delta \omega_{i}(n)=v \beta \Delta G_{i} / 2
$$

with $\beta$ being the linewidth enhancement factor and $\Delta G_{i}$ the change in the modal gain with respect to its threshold value. 
Equation (2) states that the carrier density increases by current injection and decreases by stimulated emission into the laser mode and into the signal mode. Equations (3) and (4) account for the changes in the complex electric field due to the stimulated emission, the cavity (losses and phase shift) and external injection.

\section{Complete Model}

A system of several coupled lasers can be described by matrix equations that are a direct extension to (2)-(4). These matrix equations are the same as (2)-(4) when the variables $n, E_{L}, E_{s}$ and the structure and material parameters are replaced with vectors of the form $\mathbf{n}=\left(n_{1} n_{2} \ldots n_{N}\right)^{T}$, where $n_{i}$ are the variable values in the individual lasers.

Of special concern is the input vector $\mathbf{E}_{\mathrm{s}}^{\text {ext }}$, which defines the coupling between the lasers, the coupling of the input signal and the bias fields of the lasers. It is written as

$$
\mathbf{E}_{\mathbf{s}}^{\text {ext }}=\sqrt{T_{L} T_{s}} \mathbf{A E}_{\mathbf{L}}+\sqrt{T_{s}} \mathbf{E}_{\text {bias }}+\sqrt{T_{s}} \mathbf{B} E_{\text {signal }}
$$

where $T_{L}$ and $T_{s}$ are the effective cavity facet transmission coefficients for optical power at the laser and signal mode frequencies, $\mathbf{A}$ is the matrix of waveguide transmission coefficients coupling the electric field of the previous laser to the next, and $\mathbf{B}$ is the vector describing how the input signal is coupled to the lasers. Every element of the input vector should satisfy $\left|E_{s}^{\text {ext }}\right| \lesssim 0.9 \Delta g L E_{s}^{\max }$ to ensure that the lasers remain unsaturated. Here $\Delta g=\alpha_{s}-G_{s}$ is the total loss for the signal mode and $E_{s}^{\max }$ is the electric field $E_{s}$ of a single laser at the saturation limit where $E_{L}=0$.

\section{STRUCTURE OF THE REGENERATOR}

A function fulfilling the requirements of a regenerator can be found using the steady-state relation (1). The optimization of the structure is nontrivial and the structure used in the calculations is found by experimentation. Therefore, the structure is not necessarily optimal, but still adequate to demonstrate the operation. Using a normalization where the input signal power at which the laser saturates is set to one, the regenerator function to implement reads as (8), shown at the bottom of the page, where the real parts $\Re$ of the square roots provide correct saturation behavior.

The above function with the electric fields represented in the power domain is plotted in Fig. 2, with a different normalization (see the figure caption for details). The peak in the output power just before saturation is due to the strong nonlinearities of the field in this area. With different coupling parameters it might be possible to reduce their magnitude, but in any case the device is never intended to enter the nearby saturation region.

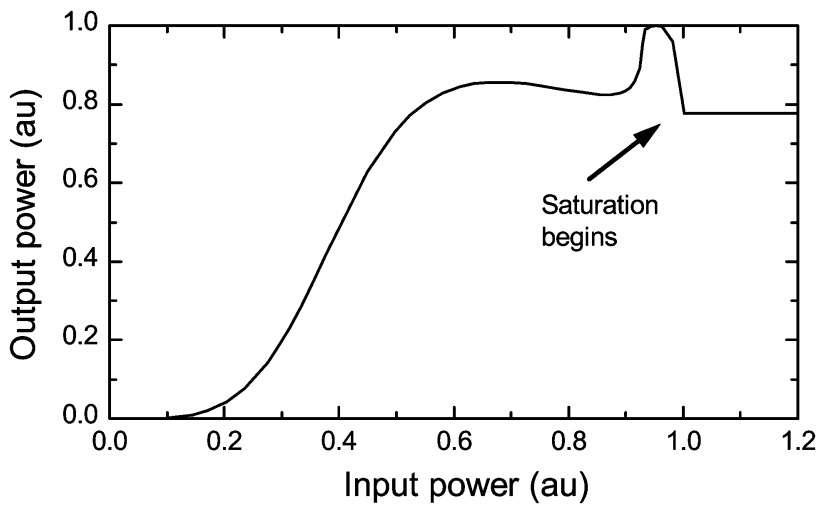

Fig. 2. Steady-state input and output powers of the regenerator, as predicted by (8). The input power is normalized with respect to the saturation power of the input, and the output with respect to the maximum output power.

The regenerator function of (8) can be implemented with a structure composed of eight lasers, band pass filters, two coherent sources to bias the lasers and optical isolators to prevent backward propagation of the signals. In principle optical isolators may not be strictly necessary. They could be replaced by active interferometric structures that effectively cancel the backward reflected amplified signal while allowing transmission and thus remove the backward coupling of the lasers, much like the isolators. Ideal optical isolators are, however, used throughout the paper, since their operation is easier to understand. The schematic configuration of the regenerator was shown in Fig. 1.

The physical operation modeled by (8) can be described approximately as follows. Term $a$ in (8) realizes a smooth absolute value -like function (formed by lasers 1 and 3 in Fig. 1) and term $b$ forms a linear function (lasers 2 and 4 ). The sum of these (interferometric junction in the middle of the device) gives a function that is roughly constant for negative values and linear for positive values. Lasers 5-8 operate in a similar manner, but their response for positive values is constant and linear for negative values. By properly scaling and shifting the output of lasers $1-4$ and feeding it to lasers 5-8 one can create a function similar to the one shown in Fig. 2. The linear function described by term $b$ could be replaced by a simple waveguide, if only steady-state operation was to be considered. For transient signals, however, the extremely fast response of linear waveguides would cause linear high frequency response and consequently bad transient regeneration characteristics. Another possibility is to use appropriately tailored resonant cavities that mimic the rise time of the signal in the nonlinear paths. This approach would be simpler to process, since it would reduce the number of lasers from eight to four.

$$
\begin{aligned}
y= & -1.744-1.8 \Re \sqrt{1-\left(0.3-1.2 \Re \sqrt{-(2.97-1.0588 a-3.0494 b)^{2}+1}\right)^{2}} \\
& +5.184 \Re \sqrt{1-\left(0.3-1.2 \Re \sqrt{-(0.628-0.4235 a-1.2198 b)^{2}+1}\right)^{2}} \\
a= & \Re \sqrt{1-\left(0.3-1.2 \Re\left(\sqrt{1-(x-0.8)^{2}}\right)\right)^{2}} \\
b= & \Re \sqrt{1-\left(0.3-1.2 \Re\left(\sqrt{1-(-0.4 x-0.24)^{2}}\right)\right)^{2}}
\end{aligned}
$$


The coupling matrix A of (7) required to implement the function $y$ in (8) can be written as $\mathbf{A}=$ $L \Delta g \mathbf{A}^{\prime} E_{s}^{\max } /\left(E_{L}^{\max } \sqrt{T_{L} T_{s}}\right)$, where $E_{L}^{\max }$ is the value of the electric field of the laser mode when $E_{s}=0$ and $\mathbf{A}^{\prime}$ is normalized similar to (8) and can be read directly from it. $\mathbf{A}^{\prime}$ is shown in (9) at the bottom of the page. If $\mathbf{A}^{\prime}$ satisfies $\Delta g<\sqrt{T_{L} T_{s}} / L / \operatorname{Max}\left\{\mathbf{A}^{\prime}\right\}$, then $\operatorname{Max}\{\mathbf{A}\}<1$ and all the waveguides are passive. For fast operation it is necessary for $\Delta g$ to have a large value, $\gtrsim 5000 / \mathrm{m}$. This dictates that the laser must be short, $\sim 10 \mu \mathrm{m}$ (the mode separation of the laser then becomes large, $\sim 18 \mathrm{meV}$, or $4.4 \mathrm{THz}$ ). The maximum value of $\Delta g$ can also be increased further by introducing gain in the waveguides connecting the lasers. In this work most of the waveguides have small gain to support the large $\Delta g$ required for fast operation.

The bias field vector in (7) is $\mathbf{E}_{\text {bias }}=E_{s}^{\max } L \Delta g \mathbf{E}_{\mathbf{b}}^{\prime} / \sqrt{T_{s}}$, where $\mathbf{E}_{\mathrm{b}}^{\prime}$ is the normalized bias vector which can again be found from (8)

$$
\mathbf{E}_{\mathbf{b}^{\prime}}=-\left[\begin{array}{llllllll}
-.8 & -.24 & .3 & .3 & 2.97 & .627 & .3 & .3
\end{array}\right]^{T} \text {. }
$$

And finally, the signal coupling vector $\mathbf{B}=$ $\mathbf{B}^{\prime} E_{s}^{\max } \Delta g L / \sqrt{T_{s}}$, is read from (8) and written as

$$
\mathbf{B}^{\prime}=\left[\begin{array}{llllllll}
1 & -.4 & 0 & 0 & 0 & 0 & 0 & 0
\end{array}\right]^{T}
$$

Now the output is available as the linear combination $E_{\text {out }}=$ $-1.74 E_{s}^{\max }+\Delta g L E_{s}^{\max }\left(-1.8 E_{L, 7}+5.18 E_{L, 8}\right) / E_{L}^{\max }$.

The values of the other parameters used in the calculation are collected in Table I. Some of the chosen values, are discussed in detail below. For high-speed operation, the length of the laser cavity should be made small. To keep the mirror losses at a reasonable level, the order of magnitude of the mirror reflectivity for the laser mode needs be $\gtrsim 0.9$. High-speed operation also determines $\Delta g, \alpha_{s}$, and reflectivities for the signal mode.

To obtain relaxation oscillation free behavior the product of the photon density and the differential gain must be high (see the Appendix). Achieving high enough values of differential gain using quantum wells as active material is at the limit of feasibility using present semiconductor materials. In contrast, for quantum dots differential gain values up to $\sim 10^{-12} / \mathrm{cm}^{2}$ have been reported [10]-[16]. In this work the material differential gain is defined by

$$
g^{\prime}=\frac{d g_{\mathrm{mat}}(n)}{d n}
$$

where $g_{\text {mat }}$ is the material gain (the gain in the case when all the photons are assumed to be confined in the active material)

\begin{tabular}{|c|c|c|}
\hline $\bar{I}$ & $5.1 \mathrm{~mA}$ & Effective injection current \\
\hline$q$ & $1.6022 \times 10^{-19} \mathrm{C}$ & Elementary charge \\
\hline$V$ & $0.3 \mu \mathrm{m} \times 3 \mu \mathrm{m} \times \mathrm{L}$ & The optical volume of the laser \\
\hline$\tau$ & $1 \mathrm{~ns}$ & $\begin{array}{l}\text { Carrier lifetime without stimulated } \\
\text { emission }\end{array}$ \\
\hline$v$ & $1 \times 10^{8} \mathrm{~m} / \mathrm{s}$ & Speed of light in the medium \\
\hline$\xi$ & $\sqrt{\varepsilon \mu^{-1}} /(\hbar \omega v)$ & $\begin{array}{l}\text { Conversion factor between }|E|^{2} \\
\text { and photon density }\end{array}$ \\
\hline$\alpha_{L}$ & $5129 / \mathrm{m}$ & Cavity loss for the laser mode \\
\hline$L$ & $10 \mu \mathrm{m}$ & Cavity length \\
\hline$\alpha_{s}$ & $20129 / \mathrm{m}$ & Cavity loss for the signal mode \\
\hline$\varepsilon$ & $9 \varepsilon_{0}$ & Permittivity of the media \\
\hline$\mu$ & $\mu_{0}$ & Susceptibility of the media \\
\hline$\hbar \omega$ & $1 \mathrm{eV}$ & Photon energy \\
\hline$n_{i}^{0}$ & $1 \times 10^{21} / \mathrm{m}^{3}$ & $\begin{array}{l}\text { Transparency carrier density } \\
\text { for modes } i \in\{L, s\}\end{array}$ \\
\hline$\beta$ & 0.1 & Linewidth enhancement factor \\
\hline$G_{i}^{\max }$ & $10000 / \mathrm{m}$ & $\begin{array}{l}\text { Maximum available modal gain } \\
\text { for modes } i \in\{L, s\}\end{array}$ \\
\hline$\Delta g$ & $\alpha_{s}-G_{s}(n)$ & Total loss of the signal mode \\
\hline$T_{s}$ & 0.182 & $\begin{array}{l}\text { Laser facet transmission coefficient } \\
\text { for optical power at signal frequency }\end{array}$ \\
\hline$T_{L}$ & 0.05 & $\begin{array}{l}\text { Laser facet transmission coefficient } \\
\text { for optical power at laser frequency }\end{array}$ \\
\hline$E_{e x t}^{L}$ & $\sqrt{10^{-7}} E_{L}^{\max }$ & Electric field for phase locking \\
\hline
\end{tabular}

TABLE I

RATE EQUATION PARAMETERS

and $n$ is the carrier density normalized to the active material volume. The modal differential gain (the carrier density normalized to the volume of the optical cavity like in this paper) is approximately equal to the material differential gain. Some uncertainty is left in the reported values of the differential gain in some of the above references due to the lack of a detailed definition. The relaxation oscillation free behavior is an essential part of the high-speed operation of the regenerator. Suppression of the relaxation oscillations requires the cavity loss to be related to the photon density and the differential gain according to $\left|E_{L}\right| \gtrsim 2 \sqrt{\left(\alpha_{L} / \gamma_{L} \xi\right)}$ (see the Appendix). In the gain model used here, the differential gain is determined by the transparency current $n_{0}$ and the maximum modal gain $g_{\max }$. On the basis of the above considerations, the parameters are set to $n_{0}=1 \times 10^{21} \mathrm{~m}^{-3}$ and $g_{\max }=10000 / \mathrm{m}$, which imply a differential gain of $1.2 \times 10^{-14} / \mathrm{cm}^{2}$ at the lasing threshold.

The last parameter of special interest is the linewidth enhancement factor. In the literature extremely low values of the linewidth enhancement factor $(\mathrm{LEF}) \sim 0.1$ have been reported [17], and there are also indications that the value zero might also be achievable, even when the effect of higher lying states is taken into consideration [18]. Here, the very low value of $\mathrm{LEF}=0.1$ is adopted.

$$
\mathbf{A}^{\prime}=\left[\begin{array}{cccccccc}
0 & 0 & 0 & 0 & 0 & 0 & 0 & 0 \\
0 & 0 & 0 & 0 & 0 & 0 & 0 & 0 \\
-1.2 & 0 & 0 & 0 & 0 & 0 & 0 & 0 \\
0 & -1.2 & 0 & 0 & 0 & 0 & 0 & 0 \\
0 & 0 & -1.059 & -3.049 & 0 & 0 & 0 & 0 \\
0 & 0 & -0.424 & -1.22 & 0 & 0 & 0 & 0 \\
0 & 0 & 0 & 0 & -1.2 & 0 & 0 & 0 \\
0 & 0 & 0 & 0 & 0 & -1.2 & 0 & 0
\end{array}\right]
$$



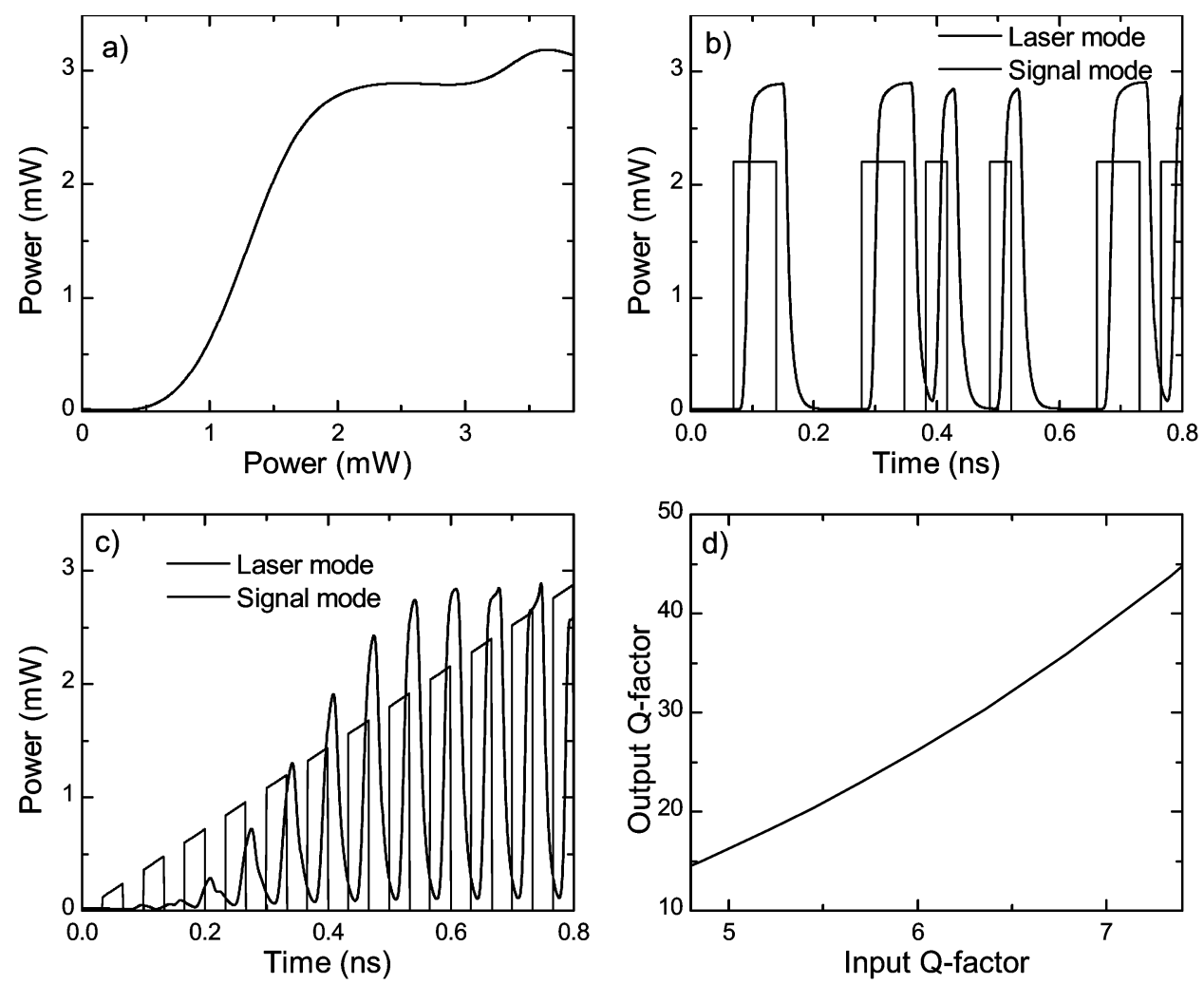

Fig. 3. Output power and regenerator characteristics of the regenerator. (a) Output power of the regenerator as a function of input power. (b) Response of the regenerator to a $30-\mathrm{GHz}$ random bit sequence (the input bits are shown in light gray). (c) Response to a 30-GHz alternating sequence of zeros and ones with the power level of one increasing linearly with time. (d) $Q$-factor of the regenerator with respect to the $Q$-factor of a normally distributed signal at the input [the calculation is based on the results shown in Fig. 3(a)]).

\section{RESUlTS AND DisCUSSION}

The coupled set of rate equations for the regenerator are solved numerically for the signals shown in Fig. 3. In Fig. 3(a) a signal with slowly increasing power is used to obtain the input-output response of the regenerator. The result is in agreement with the results from the steady-state model of (8), shown in Fig. 2. Fig. 3(b) and (c) demonstrate the transient operation when the input is stimulated by a random bit sequence at $30 \mathrm{GHz}$ [Fig 3(b)] and an alternating sequence of zeros and ones with the power level of the one increasing linearly with time [Fig. 3(c)].

The results shown in Fig. 3(a) are then used to evaluate the $Q$-factor at the output with respect to the $Q$-factor at the input. Calculation is done for a non return to zero modulated signal with power levels $0 \mathrm{~mW}$ for zero and $2.9 \mathrm{~mW}$ for one. $Q$-factor is defined as [19]

$$
Q=\frac{y_{1}-y_{0}}{\sigma_{0}+\sigma_{1}}
$$

where $y_{0}, y_{1}, \sigma_{0}$, and $\sigma_{0}$ are the means and standard deviations of the zero and one levels, respectively. In the calculation the regenerator is treated deterministically, and the probability density of the output is written as

$$
p(y)=\sum_{i=1}^{j} \frac{p_{x}\left(f_{i}^{-1}(y)\right)}{\left|f^{\prime}(y)\right|} .
$$

Here $p_{x}(x)$ is the probability distribution of the input signal, $f(x)$ is the response of the system to input $x$, and $i$ goes over the different components of $f^{-1}(y)$ in case $f$ is not monotonous and $f^{-1}(y)$ has $j$ ambiguous points. For simplicity a gaussian input distribution centered at $2.9 \mathrm{~mW}$ is used for the logical one and the positive half of the gaussian distribution centered at 0 for the logical zero (phase fluctuations of the signal are thus not taken into account, which is a reasonable approximation, because the input signal is assumed to be locked to the same phase with the rest of the system). The results of the calculation are shown in Fig. 3(d), which shows that $Q$-factor is strongly enhanced.

The bit-error rate (BER) of a Gaussian signal is strongly dependent on the $Q$-factor (for $Q=6 \mathrm{BER} \approx 10^{-9}$ and for $Q=7 \mathrm{BER} \approx 1.3 \times 10^{-12}$ ). Note, however, that the reduction of the $Q$-factor by the regenerator does not reduce BER. It merely states that the noise distribution has become narrower and thus more tolerant to additional noise. In addition to the input and output, there are 16 other electric field and eight carrier density variables to track. It is meaningful to present only an example of them here, since their general behavior is very alike, and their exact temporal behavior with respect to the input signal is not very interesting.

Fig. 4(a) shows the electric field power in one of the lasers, for the input signal that is plotted in Fig. 2(c). The sum of the laser mode and signal mode power is constant, except for some brief moments when the laser is out of equilibrium. The peaks in the power at the laser frequency result from the relaxation of the laser to a new state, but the relaxation is monotonous 


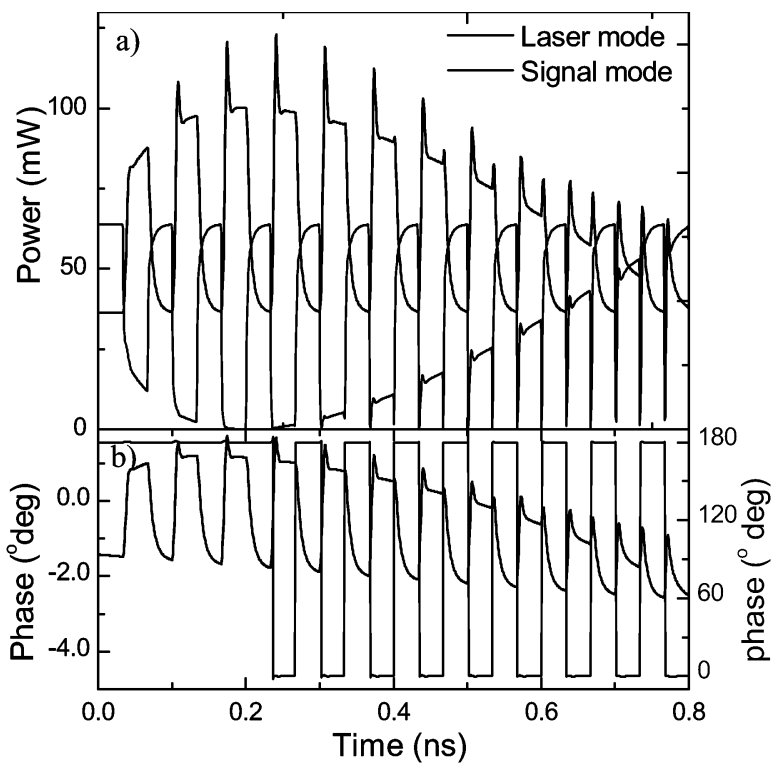

Fig. 4. (a) Powers of the electric fields inside laser 1 of Fig. 1. The black curve is for the laser mode and the grey curve for the signal mode. (b) Phase of the laser mode is plotted in black (axis to left) and the phase of the signal in grey (axis to right).

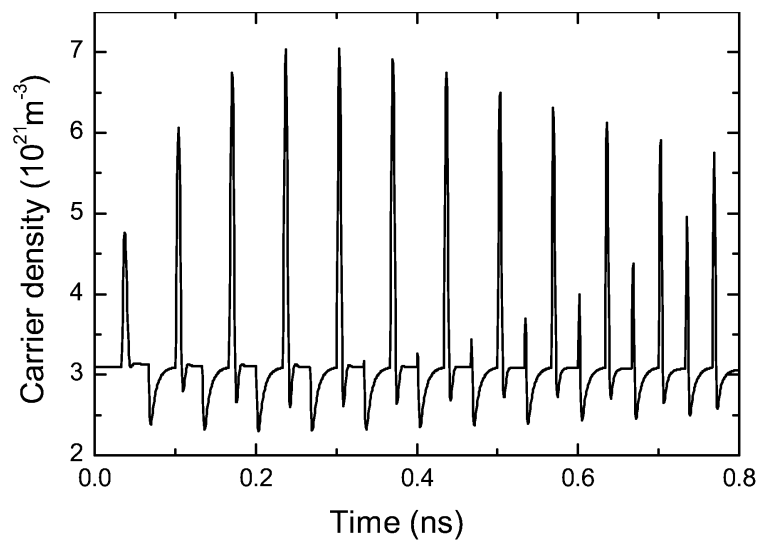

Fig. 5. Carrier density of laser 1 of Fig. 1. While the carrier density has high peak values in transient operation, it is constant when steady-state is reached.

and as such not to be confused with relaxation oscillations. The phases of the fields are shown in Fig. 4(b). The phase of the laser mode stays within a few degrees from zero because of the combined effect of phase locking and the low value of LEF. A value of LEF a few times higher is not disastrous in most cases, but the regeneration properties get worse as the LEF increases. The signal mode phase varies between 0 and 180 degrees (in and out of phase with the bias signal). Stability analysis beyond simple numerical verifications has not been done, but with any experimented steady-state input signal, the phases and magnitudes of the fields seem to converge. The corresponding carrier densities are shown in Fig. 5.

To give an idea of how the operation would change if relaxation oscillations were present, an example of the output with strong relaxation oscillations is plotted (Fig. 6). This plot is obtained by solving the problem as above, but with the output

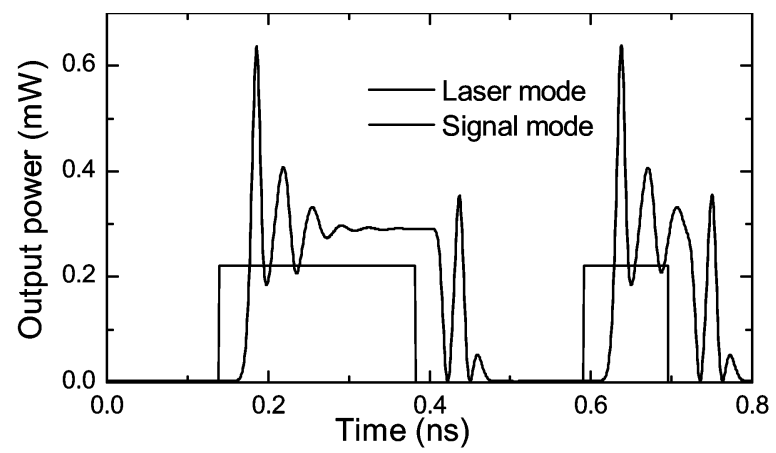

Fig. 6. If the injection current of the lasers is sufficiently dropped, the relaxation oscillations are no longer suppressed, as seen in the figure.

power dropped by a factor of ten. A similar effect could be obtained by reducing the differential gain of the laser.

The most important features excluded from the present model are proper internal noise treatment and the absence of gain saturation. Also the phase locking of the master laser to the input phase is assumed ideal. These should be included in a more complete model. Note that the carrier density is approximately constant in steady state and that the nonradiative lifetime of carriers is always large compared to the radiative lifetime. Therefore, the carrier density independence of the lifetime is not considered critical in the present model.

The most important artifact introduced by noise would be the slow drifting of the laser phase. In comparison to the drifting caused by the changes in the carrier density and cavity resonance, the effect should prove to be negligible and eliminated by the phase locking. The effect of the distributed noise sources (spontaneous emission in each laser) is harder to estimate without exact calculations. The gain saturation is not likely to be an essential effect, but only modifies how the parameter values should be chosen.

In the present model, the natural cavity resonances are not fine tuned to compensate for the average of the variations caused by carrier density fluctuations. This could add some tolerance to larger values of LEF, and reduce the deviation of steady-state laser phase from zero. Under certain circumstances, it should also be possible to compensate for larger values of LEF by changing the phase shifts of the connecting waveguides.

The parameters and the structure of the regenerator have been chosen to be as realistic as possible to demonstrate the device properties. Although integrated optical isolators have been manufactured [20], [21], isolators suitable for the purposes of the device may not be easily constructed. Therefore, using active anti reflectors, described only shortly above, as well as replacing four of the lasers with passive cavities, are interesting possibilities. Also meeting the requirements for some device parameters is at the limit of technological feasibility, so far. The discussion about some of the more or less subtle means of enhancing or modifying the operation by the antireflectors, feedback, and compensating for the effects of LEF by phase shifted waveguides is out of scope of this manuscript and postponed to a later work. 


\section{CONCLUSION}

A $2 \mathrm{R}$ regenerator is demonstrated using a novel scheme to realize arbitrary functions using purely optical coherent signals. The method relies on the use of phase-locked laser amplifiers, optical isolators and coupled waveguides.

The operation speed of the demonstrated regenerator can be adjusted with the device parameters and according to small signal analysis, speeds well in excess of $100 \mathrm{GHz}$ might be possible. The fast operation is based partly on the fact that the lasers of the system never saturate, like in other demonstrated semiconductor regenerators, and that the carrier lifetime is very short because of the ever present large photon density.

Basic coherent optical devices like phase-locked lasers have been studied extensively, but little attention has been paid to more complex structures exploiting coherence. Although technologically challenging, the device concept studied here offers means to circumvent some of the problems limiting the operation speed found in conventional active devices. In addition it enables generating arbitrary functions in the optical domain, that could be used for purely optical logic, memory elements and re-amplifying, re-shaping, and re-timing (3R) regeneration.

\section{APPENDIX}

In this Appendix, it is shown that it is possible to find an operating point where relaxation oscillations no longer take place. The connection to the maximum operating frequency is also discussed.

In small signal analysis the linearized variables $n=$ $n_{0}+n_{\Delta}, G_{L}=G_{L 0}+\gamma_{L} n_{\Delta}, E_{L}=E_{L 0}+E_{L \Delta}, E_{L}^{\mathrm{ext}}=$ $E_{L 0}^{\mathrm{ext}}, G_{s}=G_{s 0}+\gamma_{s} n_{\Delta}, E_{s}=E_{s 0}+E_{s \Delta}, E_{s}^{\mathrm{ext}}=E_{s 0}^{\mathrm{ext}}+E_{s \Delta}^{\mathrm{ext}}$ are substituted in (2)-(4). In these variables the index 0 refers to the steady-state value of the variable and index $\Delta$ to the small deviation from the steady-state value. The modal differential gain is denoted by $\gamma$. In addition to these, substitutions $G_{L 0}=\alpha_{L}, G_{s 0}=\alpha_{s}-\Delta g, \Delta \omega_{i}=0$ and $E_{L 0}^{\text {ext }}=0$ are used. Using the steady-state equations, neglecting terms of order higher than 1 and taking the Laplace transform gives

$$
\begin{aligned}
s n_{\Delta}= & -\left(v \xi \gamma_{L} E_{L 0}^{2}+v \xi \gamma_{s} E_{s 0}^{2}+\tau^{-1}\right) n_{\Delta} \\
& -2 v \xi \alpha_{L} E_{L 0} E_{L \Delta}-2 v \xi G_{s 0} E_{s 0} E_{s \Delta} \\
s E_{L \Delta}= & \frac{1}{2} v \gamma_{L} E_{L 0} n_{\Delta} \\
s E_{s \Delta}= & \frac{1}{2} v \gamma_{s} n_{\Delta} E_{s 0}-\frac{1}{2} v \Delta g E_{s \Delta}+\mathcal{L}\left\{E_{s \Delta}^{\mathrm{ext}}\right\}
\end{aligned}
$$

where $\mathcal{L}$ is the Laplace transform operator and $s$ the Laplace transform variable.
The solutions are essentially of the form $p_{2} / p_{3}$, where $p_{2}$ is a polynomial of the second order or lower, and $p_{3}$ is the characteristic polynom of the problem

$$
\begin{aligned}
p_{3}= & 2 s^{3} \tau+v^{3} \xi \alpha_{L} E_{L 0}^{2} \Delta g \gamma_{L} \tau \\
& +\left(v \Delta g \tau+2+2 v \xi \tau \gamma_{L} E_{L 0}^{2}+2 v \xi \tau \gamma_{s} E_{s 0}^{2}\right) s^{2} \\
& +\left(2 v^{2} \xi \alpha_{L} E_{L 0}^{2} \gamma_{L} \tau+v \Delta g+v^{2} \Delta g \xi \tau \gamma_{L} E_{L 0}^{2}\right. \\
& \left.-v^{2} \Delta g \xi \tau \gamma_{s} E_{s 0}^{2}+2 v^{2} \xi \tau E_{s 0}^{2} \gamma_{s} \alpha_{s}\right) s .
\end{aligned}
$$

The characteristic polynom factorizes at points $E_{s 0}=0$ and $E_{L 0}=0$

$$
\begin{aligned}
p_{3}^{E_{s}=0}= & (2 s+v \Delta g) \\
& \left(s^{2} \tau+s+s v \xi \tau \gamma_{L} E_{L 0}^{2}+v^{2} \xi \alpha_{L} E_{L 0}^{2} \gamma_{L} \tau\right) \\
p_{3}^{E_{L}=0}= & s\left(2 s^{2} \tau+s v \Delta g \tau+2 s+2 s v E_{s 0}^{2} \xi d G_{s} \tau\right. \\
& \left.-v^{2} \gamma_{s} E_{s 0}^{2} \xi \tau \Delta g+2 v^{2} \gamma_{s} E_{s 0}^{2} \xi \tau \alpha_{s}+v \Delta g\right)
\end{aligned}
$$

The relaxation oscillations do not exist when the roots of the characteristic polynom are real. This is equal to requiring the discriminants of the second order terms in the factorized polynoms be positive. These conditions give (21) and (22), shown at the bottom of the page, where $N_{\mathrm{ph}}^{\mathrm{tot}}=\xi\left(E_{L 0}^{2}+E_{s 0}^{2}\right)$ is the total photon density of the cavity.

Meeting these conditions at $E_{s 0}=0$ and $E_{L 0}=0$ does not guarantee nonoscillatory behavior for all intermediate operating points and parameters values. However, for realistic parameter values oscillatory solutions should not be present if both limits are met.

A widely used model to describe the relaxation oscillation frequency $f_{\mathrm{RO}}$ in semiconductor laser relates it to the photon density of the cavity $N_{\mathrm{ph}}$ as $f_{\mathrm{RO}}^{2} \sim N_{\mathrm{ph}}$ [22]-[25]. This model is based on solving the small signal approximation of the rate equations and neglecting some higher order terms that are insignificant for low photon densities. More accurate treatment results in $f_{\mathrm{RO}}^{2} \sim N_{\mathrm{ph}}-C_{1} N_{\mathrm{ph}}^{2}-C_{2}$, where relaxation oscillations are completely suppressed when the frequency becomes imaginary [26]. ${ }^{1}$ The more accurate model and the model presented here thus allow suppressing relaxation oscillations completely.

The time constants of the small signal system can also be determined from the poles of the characteristic polynom. As the exact form of the poles is known only for the two limiting points, the location of the first order pole at the operating point closest to saturation, $E_{s}=0.9 E_{s}^{\max }$, can be interpolated to $\sim v \Delta g / 20$. The poles of the second order terms at the two limits suggest that the corresponding time constants are fairly unsignificant in comparison to the first order time constant, for most realistic parameter combinations. Therefore, the operation speed is well approximated by the first order term, under these conditions.

${ }^{1}$ This can also be easily found by a direct calculation similar to the one presented in the Appendix.

$$
\begin{aligned}
& N_{\mathrm{ph}}^{\mathrm{tot}}>\frac{-1+2 \tau v \alpha_{L}+2 \sqrt{-\tau v \alpha_{L}+\tau^{2} v^{2} \alpha_{L}^{2}}}{v \tau \gamma_{L}} \approx 4 \frac{\alpha_{L}}{\gamma_{L}} \\
& N_{\mathrm{ph}}^{\mathrm{tot}}>\frac{-3 v \Delta g \tau+4 v \tau \alpha_{s}-2+2 \sqrt{2} \sqrt{v \tau\left(-\Delta g+\alpha_{s}\right)\left(-v \Delta g \tau+2 v \tau \alpha_{s}-2\right)}}{2 v \tau \gamma_{s}}
\end{aligned}
$$


Considering the possible values of the dominating pole, bandwidths in excess of $100 \mathrm{GHz}$ should be attainable for optimized material and device parameters.

\section{REFERENCES}

[1] O. Leclerc, B. Lavigne, E. Balmefrezol, P. Brindel, L. Pierre, D. Rouvillain, and F. Sequineau, "Optical regeneration at $40 \mathrm{~Gb} / \mathrm{s}$ and beyond," $J$. Lightw. Technol., vol. 21, no. 11, pp. 2779-2790, Nov. 2003.

[2] K. E. Stubkjaer, "Semiconductor optical amplifier-based all-optical gates for high-speed optical processing," IEEE J. Sel. Topics Quantum Electron., vol. 6, no. 6, pp. 1428-1435, Nov. 2000.

[3] G. Morthier, M. Zhao, B. Vanderhaegen, and R. Baets, "Experimental demonstration of an all-optical $2 \mathrm{R}$ regenerator with adjustable decision threshold and 'true' regeneration characteristics," IEEE Photon. Technol. Lett., vol. 12, no. 11, pp. 1516-1518, Nov. 2000.

[4] A. Kuramoto and S. Yamashita, "All-optical regeneration using a side-mode injection-locked semiconductor laser," IEEE J. Sel. Topics Quantum Electron., vol. 9, no. 5, pp. 1283-1287, Sep. 2003.

[5] S. Piazzolla, P. Spano, and M. Tamburrini, "Small signal analysis of frequency chirping in injection-locked semiconductor lasers," IEEE J. Quantum Electron., vol. 22, no. 12, pp. 2219-2223, Dec. 1986.

[6] R. Lang, "Injection locking properties of a semiconductor laser," IEEE J. Quantum Electron., vol. 18, no. 6, pp. 976-983, Jun. 1982.

[7] F. Mogensen, H. Olesen, and G. Jacobsen, "Locking conditions and stability properties for semiconductor laser with external light injection," IEEE J. Quantum Electron., vol. 21, no. 7, pp. 784-793, Jul. 1985.

[8] Y. Hong and K. A. Shore, "Locking characteristics of a side-mode injected semiconductor laser," IEEE J. Quantum Electron., vol. 35, no. 11, pp. 1713-1717, Nov. 1999.

[9] C.-H. Chang, L. Chrostowski, and C. J. Chang-Hasnain, "Injection locking of VCSEL's," IEEE J. Sel. Topics Quantum Electron., vol. 9, no. 5, pp. 1386-1393, Sep. 2003.

[10] N. Kirstaedter, O. G. Schmidt, N. N. Ledentsov, D. Bimberg, V. M. Ustinov, A. Y. Egorov, A. E. Zhukov, M. V. Maximov, and P. S. Kop'ev, "Gain and differential gain of single layer InAs/GaAs quantum dot injection lasers," Appl. Phys. Lett., vol. 69, no. 9, pp. 1226-1228, Aug. 1996.

[11] T. Riedl, A. Hangleiter, J. Porsche, and F. Scholz, "Small-signal modulation response of InP/GaInP quantum-dot lasers," Appl. Phys. Lett., vol. 80, no. 21, pp. 4015-4017, May 2002.

[12] D. Bimberg, N. Kirstaedter, N. N. Ledentsov, Z. I. Alferov, P. S. Kop'ev, and V. M. Ustinov, "InGaAs-GaAs quantum-dot lasers," IEEE J. Sel. Topics Quantum Electron., vol. 3, no. 2, pp. 196-205, Apr. 1997.

[13] K. Kamath, J. Phillips, H. Jiang, J. Singh, and P. Bhattacharya, "Smallsignal modulation and differential gain of single-mode self-organized $\mathrm{In}_{0.4} \mathrm{Ga}_{0.6} \mathrm{As} / \mathrm{GaAs}$ quantum dot lasers," Appl. Phys. Lett., vol. 70, no. 22, p. 2952, Jun. 1997.

[14] A. A. Ukhanov, A. Stintz, P. G. Eliseev, and K. J. Malloy, "Comparison of the carrier induced refractive index, gain, and linewidth enhancement factor in quantum dot and quantum well lasers," Appl. Phys. Lett., vol. 84, no. 7, pp. 1058-1060, Feb. 2004.

[15] P. Bhattacharya, S. Ghosh, S. Pradhan, J. Singh, Z.-K. Wu, J. Urayama, K. Kim, and T. B. Norris, "Carrier dynamics and high-speed modulation properties of tunnel injection InGaAs-GaAs quantum-dot lasers," IEEE J. Quantum Electron., vol. 39, no. 8, pp. 952-962, Aug. 2003.

[16] S. Ghosh, S. Pradhan, and P. Bhattacharya, "Dynamic characteristics of high-speed $\operatorname{In}_{0.4} \mathrm{Ga}_{0.6} \mathrm{As} / \mathrm{GaAs}$," Appl. Phys. Lett., vol. 81, no. 16, pp. 3055-3057, Oct. 2002.
[17] T. Newell, D. Bossert, A. Stintz, B. Fuchs, K. Malloy, and L. Lester, "Gain and linewidth enhancement factor in InAs quantum-dot laser diodes," IEEE Photon. Technol. Lett., vol. 11, no. 12, pp. 1527-1529, Dec. 1999.

[18] J. Oksanen and J. Tulkki, "Linewidth enhancement factor and chirp in quantum dot lasers," J. Appl. Phys., vol. 94, no. 3, pp. 1983-1989, Aug. 2003.

[19] T. E. Stem and K. Bala, Multiwavelength Optical Networks, a Layered Approach. Reading, MA: Addison-Wesley, 1999. was Bal99.

[20] T. Shintaku, "Integrated optical isolator based on efficient nonresiprocal radiation mode conversion," Appl. Phys. Lett., vol. 73, no. 14, pp. 1946-1948, Oct. 1998.

[21] K. Gallo, G. Assanto, K. R. Parameswaran, and M. M. Fejer, "All-optical diode in a periodically poled lithium niobate waveguide," Appl. Phys. Lett., vol. 79, no. 3, pp. 314-316, Jul. 2001.

[22] R. Olshansky, P. Hill, V. Lanzisera, and W. Powazinik, "Frequency response of $1.3 \mu \mathrm{m} \mathrm{InGaAsP} \mathrm{high} \mathrm{speed} \mathrm{semiconductor} \mathrm{lasers,"} \mathrm{IEEE} \mathrm{J.}$ Quantum Electron., vol. 23, no. 9, pp. 1410-1418, Sep. 1987.

[23] M.-H. Mao, F. Heinrichsdorf, A. Krost, and D. Bimberg, "Study of high frequency response of self-organized stacked quantum dot lasers at room temperature," Electron. Lett., vol. 33, no. 19, pp. 1641-1642, Sep. 1997.

[24] O. Kjebon, R. Shatz, S. Lourdudoss, S. Nilsson, B. Stålnacke, and L. Bäckbom, " $30 \mathrm{GHz}$ direct modulation bandwidth in detuned loaded InGaAsP DBR lasers at $1.55 \mu \mathrm{m}$ wavelength," Electron. Lett., vol. 33, no. 6, pp. 488-499, Mar. 1997.

[25] K. Y. Lau and A. Yariv, "Ultra-high speed semiconductor lasers," IEEE J. Quantum Electron., vol. 21, no. 2, pp. 121-137, Feb. 1985.

[26] R. Paiella, R. Martini, F. Capasso, C. Gmachl, H. Y. Hwang, D. L. Sivco, J. N. Baillargeon, A. Y. Cho, E. A. Whittaker, and H. C. Liu, "High-frequency modulation without the relaxation oscillation resonance in quantum cascade lasers," Appl. Phys. Lett., vol. 79, no. 16, pp. 2526-2528, Oct. 2001.

Jani Oksanen was born in Finland in 1977. He received the M.Sc. degree in electrical engineering from Helsinki University of Technology (HUT), Helsinki, Finland, in 2001. He is currently pursuing the Ph.D. degree at the Laboratory of Computational Engineering, HUT.

Jukka Tulkki received the Ph.D. degree in physics from the Helsinki University of Technology (HUT), Helsinki, Finland, in 1986. His thesis addressed inelastic resonance scattering of X-rays.

From 1985 to 1987, he was an acting Associate Professor of physics at HUT. He held research appointments at the University of Helsinki (1988), the Academy of Finland (1990-1993), and at HUT (1994-1998). Since 1998, he has been a Professor of computational engineering at HUT. He is the author or coauthor of more than 60 journal papers and numerous conference presentations. His current research interests include X-ray physics, atomic collision physics, semiconductors, electronic, and optical properties of mesoscopic systems and microsystems. 